\title{
The effect of palm oil, lard, and puff-pastry margarine on postprandial lipid and hormone responses in normal-weight and obese young women
}

\author{
Janne Jensen ${ }^{1}$, Anette Bysted ${ }^{1}$, Steen Dawids ${ }^{2}$, Kjeld Hermansen ${ }^{3}$ and Gunhild Hølmer ${ }^{1}$ \\ ${ }^{1}$ Department of Biochemistry and Nutrition, The Technical University of Denmark, Building 224, 2800 Lyngby, Denmark \\ ${ }^{2}$ Medical Clinic I, Bispebjerg Hospital, 2400 Copenhagen, Denmark \\ ${ }^{3}$ Department of Endocrinology and Metabolism, Aarhus Amtssygehus, 8000 Aarhus, Denmark
}

(Received 7 September 1998 - Revised 12 April 1999 - Accepted 31 May 1999)

\begin{abstract}
Only a few studies have been published on the postprandial effects of different fatty acids in obese subjects. Therefore, the present study investigated the effects of three test meals containing palm oil (PO), lard (LD), or puff-pastry margarine (PPM), all normal dietary ingredients, on postprandial lipid and hormone responses in normal-weight and obese young women. The study was performed as a randomized, crossover design. The fats differed in the content of palmitic acid, stearic acid, and trans monounsaturated fatty acids allowing a dietary comparison of different 'solid' fatty acids. The obese women had significantly higher fasting concentrations and postprandial responses of plasma total triacylglycerol (TAG), chylomicron-TAG, and insulin compared with the normal-weight women but there was no significant difference in the postprandial responses between the three test meals. The obese women had fasting concentrations of leptin four times greater than the normal-weight women. There were no postprandial changes in the concentrations of leptin. The fasting concentrations of HDL-cholesterol were significantly lower in the obese women than in the normal-weight women, whereas there was no significant difference between the two groups in the concentrations of total cholesterol or LDL-cholesterol. These results provide evidence that obese women have exaggerated lipid and hormone responses compared with normal-weight women but the different contents of saturated and trans monounsaturated fatty acids provided by PO, LD, and PPM have no effect in either group.
\end{abstract}

Obesity: Postprandial response: Trans fatty acids: Saturated fatty acids

The high prevalence of obesity in the Western world and its well-documented association with the cardiovascular risk factors diabetes mellitus, dyslipidaemia, and hypertension represents a major problem for the general health status of industrialized countries. Diet therapy, with an emphasis on weight control is considered the major intervention to control the metabolic imbalance and attempt to reduce the risk of accelerated atherosclerosis.

The dietary treatment of obese persons has focused on diets relatively high in carbohydrate and low in total fat. However, recent studies have reported that highcarbohydrate diets may cause aggravation of dyslipidaemia in patients with non-insulin-dependent diabetes mellitus (Garg et al. 1988, 1992; Coulston et al. 1989). Instead of reduction in total fat, several official nutritional recommendations include reduction of the intake of saturated fatty acids (SFA) which are known to increase the risk of CHD. However, not all SFA are equally cholesterolaemic. The serum cholesterol raising effect of SFA in the diet appears to be limited to lauric acid (12:0), myristic acid (14:0), and palmitic acid (16:0) while stearic acid (18:0) seems to be neutral (Hegsted et al. 1965; Grande et al. 1970; Bonanome \& Grundy, 1988; Denke \& Grundy, 1992; Storm et al. 1997).

The responses of plasma lipoproteins during absorption of dietary fats have gathered increasing interest due to the fact that man, by eating regular meals, spends most of the time in the postprandial phase. Plasma total triacylglycerols (TAG) increase shortly after the first meal of the day and return to baseline concentrations several hours after the last meal. Intestine-derived chylomicrons (CM) as well as liverderived VLDL, both of which contribute to the postprandial responses (Cohn et al. 1988a; Potts et al. 1991), compete for hydrolysis by lipoprotein lipase (EC 3.1.1.34) as well as the hepatic receptor-mediated removal of remnants from plasma (Brunzell et al. 1973; Schneeman et al. 1993). Previous studies have shown that the intensity of postprandial lipaemia, especially the presence of remnant particles, plays an

\footnotetext{
Abbreviations: AUC, area under the curve; $\mathrm{CH}$, cholesterol; CM, chylomicron; LD, lard; MUFA, monounsaturated fatty acid; PO, palm oil; PPM, puff-pastry margarine; PUFA, polyunsaturated fatty acid; SFA, saturated fatty acid; TAG, triacylglycerol.

*Corresponding author: Dr A. Bysted, fax +45 4588 6307, email bysted@ mimer.be.dtu.dk
} 
important role in the pathogenesis and progression of atherosclerosis (Groot et al. 1991; Patsch et al. 1992; Karpe et al. 1994). Delayed clearance of the TAG circulating in the blood is therefore associated with increased atherogenic risk.

The influence of trans fatty acids in the diet has been discussed during recent years. Experiments with human subjects (Mensink et al. 1992; Nestel et al. 1992; Lichtenstein et al. 1993; Wood et al. 1993; Judd et al. 1994) have pointed to an unfavourable effect of trans fatty acids on blood lipids. An epidemiological study performed by Willett et al. (1993) showed a relation between intake of trans fatty acids from partially hydrogenated vegetable oils and risk of CHD.

Several studies suggest that low-carbohydrate, high-fat diets where monounsaturated fatty acids (MUFA) constitute the majority of fat have beneficial effects for patients with non-insulin-dependent diabetes mellitus compared with high-carbohydrate diets. The beneficial effects include improved glycaemic control (Garg et al. 1988; Rasmussen et al. 1993) and a subdued $24 \mathrm{~h}$ ambulatory blood pressure (Rasmussen et al. 1993). Christiansen et al. (1997) studied the effects of diets enriched in SFA or trans MUFA on the postprandial insulinaemia and fasting serum levels of lipids and lipoproteins in obese patients with non-insulindependent diabetes mellitus. They found that in the presence of unchanged glycaemia, both dietary SFA and trans fatty acids induced an increase in postprandial insulinaemia compared with a diet with cis MUFA. However, it was impossible to differentiate precisely between a long-term effect of the different diets on postprandial insulinaemia and an acute effect of the diets following the test meal or even a combination of these effects (Christiansen et al. 1997). In this context it is interesting that recent studies with mouse islets have revealed a higher insulinotropic potency of the trans fatty acids compared with the cis forms (Alstrup et al. 1999). Information about the acute metabolic effect on lipid and hormone responses in obese subjects after consumption of different types of fat is sparse. Rasmussen et al. (1996) studied the acute metabolic effect on blood glucose and insulin responses in subjects with non-insulin-dependent diabetes mellitus after ingestion of SFA and cis MUFA and they found that SFA increased the release of insulin compared with MUFA. Ingestion of trans MUFA was not studied.

In the present investigation we compared the acute postprandial effects of three normal edible fats used for spreads and cooking, differing in contents of $16: 0,18: 0$, and trans MUFA but containing comparable amounts of $c i s$ MUFA and polyunsaturated fatty acids (PUFA), on plasma total TAG, CM-TAG, insulin, and leptin responses in normal-weight and obese young women. This is important as trans MUFA, present in food products that contain partially hydrogenated oils and ruminant fats, are often substituted for SFA due to their similar physical properties. The comparison between normal-weight and obese women is interesting since the higher TAG levels normally observed in obese subjects might be a result of delayed clearing. Previously, some studies have found that insulin has a stimulatory effect on serum leptin after $6 \mathrm{~h}$ at high physiological concentrations (Malmström et al. 1996), that leptin increases between 5 and $10 \mathrm{~h}$ after short-term overfeeding (Kolaczynski et al. 1996b; Joannic et al. 1998; Saad et al. 1998), and that changing meal time by $6.5 \mathrm{~h}$ will shift rhythm by $5-7 \mathrm{~h}$ (Schoeller et al. 1997). Taking into account that the increase in leptin levels is related to the insulin fluctuations in response to meals (Laughlin \& Yen, 1997) we wanted to see whether any increment in leptin occurred up to $8 \mathrm{~h}$ after the test meals and whether the responses differed between normal-weight and obese subjects. The fatty acid compositions of the postprandial CM lipids were studied to determine if discrimination in the absorption or a specific clearing was taking place.

\section{Experimental methods}

\section{Subjects}

Seven obese women and eight normal-weight women participated in the study. The BMI was used to group the women. The normal-weight group had BMI $19.2-23.7 \mathrm{~kg} / \mathrm{m}^{2}$ and the obese group had BMI $28 \cdot 8-47 \cdot 5 \mathrm{~kg} / \mathrm{m}^{2}$. The normalweight and obese women were well matched for age (mean 27 (SD 2) years v. 29 (SD 3) years respectively).

Two women of normal weight used oral contraceptives. Three women from the obese group were smokers. None of the women was taking any medications and none had any history of recent intercurrent diseases. There was no evidence of lipid or thyroid dysfunction or kidney and liver disease. The haemostatic variables were in the normal range.

The women in the obese group were outpatients of the medical clinic at Bispebjerg Hospital in Copenhagen, Denmark and the women in the control group were students and personnel at The Technical University of Denmark.

Participants were fully informed of the experimental nature of the study and the protocol was approved by the Municipal Ethical Committee of Copenhagen and Frederiksberg, Denmark to be in accordance with the Helsinki-II declaration.

\section{Study design}

The study was performed as a randomized, crossover design where all women consumed three test meals with palm oil (PO), lard (LD), or puff-pastry margarine (PPM). The test meals were given with a washout period of at least 1 week. After an overnight fast the participants arrived in the morning at the medical clinic where a cannula was inserted into a vein from which blood samples were collected during the day. Following the collection of a fasting blood sample the test meal was served and ingested within $15 \mathrm{~min}$. Blood samples were taken at hourly intervals including at $8 \mathrm{~h}$.

The concentrations of plasma total TAG and CM-TAG were determined before the meal and monitored over $8 \mathrm{~h}$. Insulin concentrations were determined in the fasting state and monitored until starting values were obtained again ( $4 \mathrm{~h}$ after consumption of the three test meals). The concentrations of leptin were determined before the meal and after $4 \mathrm{~h}$ and $8 \mathrm{~h}$. Fasting blood samples were also analysed to determine concentrations of total cholesterol (CH), LDL-CH, and HDL-CH. 
Table 1. Major fatty acids ( $\mathrm{g} / 100 \mathrm{~g}$ total fatty acids) in palm oil (PO), lard (LD), and puff-pastry margarine (PPM)

\begin{tabular}{|c|c|c|c|}
\hline Fatty acid & PO & LD & PPM \\
\hline $14: 0$ & $1 \cdot 1$ & 1.6 & 0.9 \\
\hline $16: 0$ & 43.5 & $25 \cdot 5$ & 34.5 \\
\hline $16: 1 n-7$ & 0.2 & $2 \cdot 2$ & $0 \cdot 1$ \\
\hline $18: 0$ & 4.6 & $16 \cdot 8$ & 7.4 \\
\hline 18:1 trans $^{*}$ & 0.2 & 0.3 & $7 \cdot 0$ \\
\hline 18:1 cis $†$ & 38.9 & $39 \cdot 2$ & 31.5 \\
\hline $18: 2 n-6$ & $10 \cdot 3$ & $10 \cdot 0$ & 14.5 \\
\hline $18: 3 n-3$ & 0.7 & $1 \cdot 2$ & $2 \cdot 1$ \\
\hline$\Sigma$ SFA & $49 \cdot 2$ & 43.9 & $42 \cdot 8$ \\
\hline ¿MUFA & 39.3 & 41.7 & $38 \cdot 6$ \\
\hline$\Sigma$ PUFA & 11.0 & 11.2 & $16 \cdot 6$ \\
\hline
\end{tabular}

SFA, saturated fatty acids; MUFA, monounsaturated fatty acids; PUFA, polyunsaturated fatty acids.

* The sum of trans isomers.

†The sum of cis isomers.

Immediately after the $4 \mathrm{~h}$ blood sample was taken an almost fat-free lunch was served as described later. Water was allowed ad libitum during the $8 \mathrm{~h}$ study period.

\section{Test meals}

Three normal edible fats with different fatty acid compositions were tested in the study. The fats were selected to allow a comparison of the effect of the two SFA, palmitic and stearic acid, and of trans MUFA. The fatty acid compositions of the three fats are given in Table 1. PO (Aarhus Oliefabrik A/S, Aarhus, Denmark) is used as an ingredient in industrially produced edible fats such as margarines. LD (Raffinol, Copenhagen, Denmark) is used for deep-fat frying, and PPM (Danisco, Grindsted, Denmark) is used for baking of Danish pastries. PO differed from LD and PPM in its high content of palmitic acid and LD differed from the other two fats in its content of stearic acid. However, the total content of 'solid' fatty acids (16:0,
18:0, and trans MUFA) was of the same magnitude. The total amount of the non-atherogenic fatty acids (cis MUFA and PUFA) was approximately the same.

The test meal consisted of mashed potato made from potato flakes (Pfanni, Pfanni-Werke, Munich, Germany), skimmed milk (MD Foods, Aarhus, Denmark ( $0 \cdot 1 \mathrm{~g}$ fat/ $100 \mathrm{~g})$ ), salt, water, and one of the three fats. The meal was served with three pieces of raw cucumber $(20 \mathrm{~g})$ and a glass of artificially-sweetened blackcurrant juice (Cadiso Food $\mathrm{A} / \mathrm{S}$, Frederikssund, Denmark). It was necessary to use $1 \mathrm{~g}$ potato flakes per $1 \mathrm{~g}$ fat to camouflage the taste and mouth feel of the fat. The water content of PPM was taken into account in the preparations.

The participants were given $29 \mathrm{~g} \mathrm{fat} / \mathrm{m}^{2}$ body surface area which gave an energy content of $1.7 \mathrm{MJ} / \mathrm{m}^{2}\left(406 \mathrm{kcal} / \mathrm{m}^{2}\right)$ of which $30 \%$ came from carbohydrate, $5 \%$ from protein, and $65 \%$ from fat. The content of fat accounted for less than $0.5 \%$ energy in the other ingredients. The average fat intake was 51 (SD 4) g per meal for the normal-weight women and 64 (SD 6) g per meal for the obese women (range 46-58g and $56-73 \mathrm{~g}$ respectively).

The nearly fat-free lunch consisted of various fruits and a piece of light crisp bread with salad and tomato. The energy content of this meal was $435 \mathrm{~kJ}$ of which $83 \%$ came from carbohydrate, $10 \%$ from protein, and $7 \%$ from fat $(<1 \mathrm{~g}$ fat). All subjects received the same amount of food for lunch.

\section{Isolation of chylomicrons}

Blood samples were taken into $10 \mathrm{ml}$ vacutainer tubes with EDTA to give a final concentration of $1 \mathrm{ml}$ EDTA/l. Plasma was separated by centrifugation $(1500 \mathrm{~g})$ for $15 \mathrm{~min}$ at room temperature. The TAG-rich $\mathrm{CM}$ were isolated by layering a $\mathrm{NaCl}$ solution of density $1.004 \mathrm{~g} / \mathrm{ml}$ on top of plasma followed by centrifugation $\left(27000 \mathrm{~g}, 1 \mathrm{~h}, 15^{\circ}\right)$ in a Sorvall centrifuge RC5C (Du Pont, Stevenage, Herts., UK) SS34 rotor. The $\mathrm{CM}$ were obtained by removing the top layer from the tube.

Table 2. Fasting and postprandial responses of plasma triacyglycerol (TAG), chylomicron triacylglycerol (CM-TAG), insulin, and leptin in normalweight and obese women consuming test meals with palm oil (PO), lard (LD), or puff-pastry margarine (PPM)*

(Mean values and pooled standard deviations for eight normal-weight and seven obese women)

\begin{tabular}{|c|c|c|c|c|c|c|c|c|c|}
\hline & \multicolumn{4}{|c|}{ Normal-weight } & \multicolumn{4}{|c|}{ Obese } & \multirow[b]{2}{*}{$\begin{array}{l}\text { Group } \\
\text { effect† }\end{array}$} \\
\hline & $\mathrm{PO}$ & LD & PPM & $\begin{array}{l}\text { Pooled } \\
\text { SD }\end{array}$ & $\mathrm{PO}$ & LD & PPM & $\begin{array}{l}\text { Pooled } \\
\text { SD }\end{array}$ & \\
\hline Fasting TAG (mmol/l) & 0.85 & 0.81 & 0.95 & 0.32 & 1.37 & 1.23 & 1.66 & 0.58 & $P<0.001$ \\
\hline Fasting CM-TAG $(\mathrm{mmol} / \mathrm{l})$ & 0.03 & 0.03 & 0.04 & 0.01 & 0.08 & 0.08 & 0.12 & 0.06 & $P<0.001$ \\
\hline TAG incremental AUC $(\mathrm{mmol} \cdot \mathrm{h} / \mathrm{l})$ & $2 \cdot 43$ & $2 \cdot 33$ & 1.99 & $1 \cdot 33$ & 3.38 & $3 \cdot 72$ & 4.23 & 1.93 & $P<0.01$ \\
\hline CM-TAG incremental AUC $(\mathrm{mmol} \cdot \mathrm{h} / \mathrm{l})$ & 1.80 & 1.32 & 1.52 & 0.71 & $2 \cdot 11$ & $2 \cdot 32$ & 2.65 & 1.09 & $P<0.01$ \\
\hline $\mathrm{TAG}_{\text {MAX }}(\mathrm{mmol} / \mathrm{l})$ & 0.51 & 0.63 & 0.58 & 0.35 & 0.77 & 0.99 & 1.05 & 0.58 & $P<0.02$ \\
\hline $\mathrm{CM}-\mathrm{TAG}_{\mathrm{MAX}}(\mathrm{mmol} / \mathrm{l})$ & 0.34 & 0.42 & 0.34 & 0.21 & 0.54 & 0.67 & 0.73 & 0.36 & $P<0.01$ \\
\hline TAG $T_{\text {MAX }}(\mathrm{h})$ & 1.75 & 1.75 & 1.88 & 0.66 & 2.14 & 1.67 & 1.57 & 0.95 & NS \\
\hline CM-TAG T & 1.88 & $2 \cdot 00$ & 2.00 & 0.58 & 1.86 & 1.67 & 1.57 & 0.92 & NS \\
\hline Fasting insulin (pmol/l) & 41 & 27 & 19 & 26 & 112 & 105 & 128 & 66 & $P<0.001$ \\
\hline Insulin incremental AÚC $(\mathrm{pmol} \cdot \mathrm{h} / \mathrm{l})$ & 168 & 209 & 213 & 115 & 749 & 500 & 512 & 501 & $P<0.001$ \\
\hline Fasting leptin (ng/ml) & 13 & 10 & 11 & 7 & 41 & 42 & 45 & 15 & $P<0.001$ \\
\hline
\end{tabular}

TAG incremental AUC, incremental area under the postprandial TAG response curve; CM-TAG incremental AUC, incremental area under the postprandial chylomicron TAG response curve; TAG ${ }_{M A X}$, maximum postprandial TAG concentration; $C M-T A G_{\text {MAX, }}$ maximum postprandial CM-TAG concentration; TAG $T_{\text {MAX, }}$ time to the first maximum postprandial TAG concentration; CM-TAG T $T_{M A X}$, time to the first maximum postprandial CM-TAG concentration; insulin incremental AUC, incremental area under the postprandial insulin response curve.

${ }^{*}$ For details of test meals and procedures see pp. 470-471.

† Group effect, differences between normal-weight and obese women tested in the split plot design as described on p. 473. 

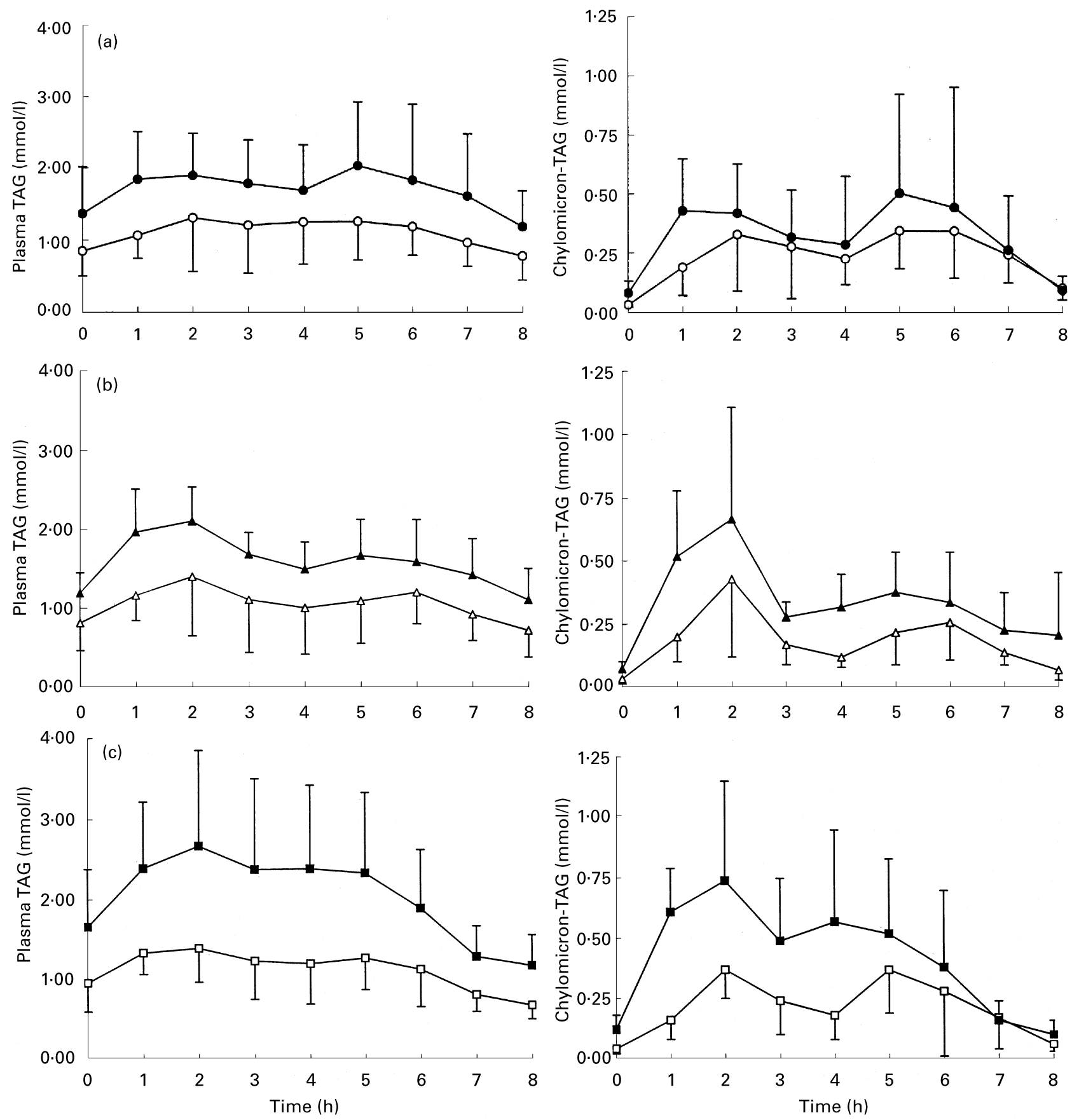

Fig. 1. Plasma triacylglycerol (TAG) and chylomicron-TAG responses $(0-8 \mathrm{~h})$ to test meals containing (a) palm oil $(\mathrm{O}, \boldsymbol{\bullet})$, (b) lard $(\triangle, \mathbf{\Delta})$, and (c) puff-pastry margarine $(\square, \mathbf{\square})$ in normal-weight $(O, \triangle, \square)$ and obese $(\boldsymbol{\bullet}, \mathbf{\Lambda}, \mathbf{\square})$ women. Values are means with standard deviations represented by vertical bars. For details of test meals and procedures see pp. 470-471.

\section{Analytical methods}

Lipids were extracted from the $\mathrm{CM}$ fractions with chloroform-methanol $(1: 1, \mathrm{v} / \mathrm{v})$, dried with $\mathrm{Na}_{2} \mathrm{SO}_{4}$, filtered and subsequently saponified with $0 \cdot 5 \mathrm{M}-\mathrm{NaOH}$ in methanol and methylated with $\mathrm{BF}_{3}$ in methanol $(140 \mathrm{~g} / \mathrm{l})$ in the presence of hydroquinone $(0.2 \mathrm{~g} / \mathrm{l}$ methanol). The fatty acid methyl esters were extracted with heptane and analysed by GLC on a Hewlett Packard 5880A instrument (Palo Alto, CA, USA) equipped with an autosampler and a flame-ionization detector. An SP2380 capillary column (30 m, i.d. $0 \cdot 32 \mathrm{~mm}$, $0 \cdot 2 \mu \mathrm{m}$ film; Supelco, Bellefonte, PA, USA) was used. The inlet pressure of the carrier gas $(\mathrm{He})$ was $76 \mathrm{kPa}$. The temperature was maintained at $250^{\circ}$ for the injector (split mode) and $260^{\circ}$ for the detector. The initial oven temperature was $140^{\circ}$. The temperature was raised to $160^{\circ}$ at $2 \% \mathrm{~min}$, then held constant for $2 \mathrm{~min}$ and finally raised $3 \% \mathrm{~min}$ to $200^{\circ}$, where it was unchanged for $5 \mathrm{~min}$.

Peaks were identified using fatty acid methyl ester standards (Nu Chek Prep, Inc., Elysian, MN, USA) and 
quantified using heptadecanoic acid (17:0) as internal standard. The major fatty acids in the isolated CM are presented as a percentage of the total fatty acid mass measured. The inter-assay CV were $<1 \%$ for major components. Previous analyses have shown that TAG is the predominant class of fatty acid-containing lipid in $\mathrm{CM}$ (> $95 \%$ ) (results not shown). As an approximation we have assumed a TAG content of $100 \%$ in CM.

Plasma total TAG and total $\mathrm{CH}$ analyses were carried out on a Boehringer Hitachi 717 EC analyser (Roche Diagnostic A/S, Hvidovre, Denmark) at $37^{\circ}$. Plasma total TAG content was measured by a standard coupled enzymic method using lipase (EC 3.1.1.3)-glycerol kinase (EC 2.7.1.30). Total CH was measured using a standard coupled enzymic procedure using cholesterol esterase (EC 3.1.1.13)-cholesterol oxidase (EC 1.1.3.17), all supplied as test kits (Boehringer Mannheim, c/o Ercopharm, Kvistgaard, Denmark). HDL-CH was determined using phosphotungstic acid $-\mathrm{MgCl}_{2}$ precipitation followed by analysis of $\mathrm{CH}$ as described earlier. LDL-CH was calculated from the measurements of total $\mathrm{CH}, \mathrm{HDL}-\mathrm{CH}$, and TAG, as described by Friedewald et al. (1972). Plasma insulin was determined by an ELISA (Andersen et al. 1993). Plasma leptin was measured by a radioimmunoassay (Maffei et al. 1995). The inter-assay CV were $1.5 \%, 1.5 \%, 2.6 \%$, $7.5 \%$ and $4.5 \%$ for plasma total TAG, total $\mathrm{CH}$, HDL-CH, plasma insulin, and plasma leptin respectively.

\section{Statistical methods}

The postprandial responses of plasma total TAG, CM-TAG, and insulin were analysed as areas under the curves (AUC) as recommended by Matthews et al. (1990). The responses were calculated separately for each subject as the difference between the absolute area and the rectangular area determined by the fasting concentrations (incremental AUC), thus representing changes occurring after the meal.

To compare the three test meals (fasting and incremental
AUC values) a split-plot structured analysis (variables: group, person, meal, period, and meal $\times$ group interaction) was carried out using the GLM procedure of the SAS System (SAS Institute Inc., Cary, NC, USA). The level of significance was set at $P<0.05$.

Repeated measures ANOVA was performed within groups to test differences in plasma total TAG, CM-TAG, insulin, and leptin levels over time.

\section{Results \\ Plasma total triacylglycerol and chylomicron-triacylglycerol}

Table 2 presents the fasting concentrations and postprandial responses of TAG in plasma and CM in normal-weight and obese women. The fasting concentrations of plasma total TAG were significantly higher in the obese women than in the normal-weight women $(P<0 \cdot 001)$. The small fractions of $\mathrm{CM}$ present in the fasting state were also isolated and the corresponding concentrations of CM-TAG were significantly higher in the obese subjects than in the normal-weight subjects $(P<0.001)$. The concentrations of plasma total TAG increased significantly during the postprandial period (Fig. 1). Some individuals showed a monophasic or a triphasic response curve but the majority showed a biphasic response curve with an initial peak $1-2 \mathrm{~h}$ and a second peak $4-7 \mathrm{~h}$ after the meal. The concentrations returned to baseline values at $8 \mathrm{~h}$. There was a parallel and significant rise in CM-TAG corresponding to the rise in plasma total TAG (Fig. 1).

The incremental areas under the postprandial plasma total TAG response curves (TAG incremental AUC) and the postprandial CM-TAG response curves (CM-TAG incremental AUC) are presented in Table 2. They were both significantly higher in the obese women compared with the normal-weight women $(P<0 \cdot 01, P<0 \cdot 01$ respectively). For TAG incremental AUC the difference was more pronounced

Table 3. Major fatty acids in palm oil (PO) and chylomicrons isolated every second hour in the postprandial period ( $\mathrm{g} / 100 \mathrm{~g}$ total fatty acids)§ (Mean values and standard deviations for eight normal-weight and seven obese women)

\begin{tabular}{|c|c|c|c|c|c|c|c|c|c|c|c|c|}
\hline \multirow[b]{2}{*}{$\begin{array}{l}\text { Time (h) ... } \\
\text { Fatty acid }\end{array}$} & & \multirow[b]{2}{*}{$\mathrm{PO}$} & \multicolumn{5}{|c|}{ Normal-weight } & \multicolumn{5}{|c|}{ Obese } \\
\hline & & & 0 & 2 & 4 & 6 & 8 & 0 & 2 & 4 & 6 & 8 \\
\hline $16: 0$ & $\begin{array}{l}\text { Mean } \\
\text { SD }\end{array}$ & 43.5 & $\begin{array}{r}25 \cdot 2 \\
2 \cdot 1\end{array}$ & $\begin{array}{r}36.6 \\
2.6\end{array}$ & $\begin{array}{r}35.7 \\
2.0\end{array}$ & $\begin{array}{r}36.0 \\
1.2\end{array}$ & $\begin{array}{r}33.0 \\
4.7\end{array}$ & $\begin{array}{r}22.5 \\
2.8\end{array}$ & $\begin{array}{r}34.5 \\
1.7\end{array}$ & $\begin{array}{r}32.6 \\
3.0\end{array}$ & $\begin{array}{r}34.5 \\
2.1\end{array}$ & $\begin{array}{r}30.5 \\
1.9\end{array}$ \\
\hline $16: 1 n-7$ & $\begin{array}{l}\text { Mean } \\
\text { SD }\end{array}$ & 0.2 & $\begin{array}{l}2 \cdot 0 \\
0.6\end{array}$ & $\begin{array}{l}0.6 \\
0.2\end{array}$ & $\begin{array}{l}0.6 \\
0.4\end{array}$ & $\begin{array}{l}0.5 \\
0.2\end{array}$ & $\begin{array}{l}0.8 \\
0.3\end{array}$ & $\begin{array}{l}2 \cdot 3 \\
1.0\end{array}$ & $\begin{array}{l}1.1 \\
0.7\end{array}$ & $\begin{array}{l}1.2 \\
0.6\end{array}$ & $\begin{array}{l}0.9 \\
0.3\end{array}$ & $\begin{array}{l}1.4 \\
0.5\end{array}$ \\
\hline $18: 0$ & $\begin{array}{l}\text { Mean } \\
\text { SD }\end{array}$ & 4.6 & $\begin{array}{r}11.3 \\
2.7\end{array}$ & $\begin{array}{l}6 \cdot 3 \\
0.9\end{array}$ & $\begin{array}{l}6.5 \\
0.8\end{array}$ & $\begin{array}{l}6 \cdot 1 \\
0.5\end{array}$ & $\begin{array}{l}7.2 \\
1.3\end{array}$ & $\begin{array}{l}7.9^{*} \\
1.3\end{array}$ & $\begin{array}{l}5.8 \\
0.2\end{array}$ & $\begin{array}{l}6 \cdot 4 \\
0.8\end{array}$ & $\begin{array}{l}5.5 \\
0.3\end{array}$ & $\begin{array}{l}6 \cdot 4 \\
0.5\end{array}$ \\
\hline $18: 1$ trans $\dagger$ & $\begin{array}{l}\text { Mean } \\
\text { SD }\end{array}$ & 0.2 & $\begin{array}{l}0.8 \\
0.2\end{array}$ & $\begin{array}{l}0.3 \\
0.1\end{array}$ & $\begin{array}{l}0.3 \\
0.1\end{array}$ & $\begin{array}{l}0.3 \\
0.1\end{array}$ & $\begin{array}{l}0.5 \\
0.2\end{array}$ & $\begin{array}{l}1 \cdot 6 \\
2 \cdot 1\end{array}$ & $\begin{array}{l}0.5 \\
0.3\end{array}$ & $\begin{array}{l}0.5 \\
0.4\end{array}$ & $\begin{array}{l}0.4 \\
0.2\end{array}$ & $\begin{array}{l}0.5 \\
0.1\end{array}$ \\
\hline $18: 1$ cis $\ddagger$ & $\begin{array}{l}\text { Mean } \\
\text { SD }\end{array}$ & 38.9 & $\begin{array}{r}26 \cdot 7 \\
1.9\end{array}$ & $\begin{array}{r}36 \cdot 8 \\
1.8\end{array}$ & $\begin{array}{r}37.1 \\
1.2\end{array}$ & $\begin{array}{r}37.4 \\
1.4\end{array}$ & $\begin{array}{r}32.4 \\
4.9\end{array}$ & $\begin{array}{c}32 \cdot 5^{\star \star} \\
3.5\end{array}$ & $\begin{array}{r}38.0 \\
1.6\end{array}$ & $\begin{array}{r}35.9 \\
1.8\end{array}$ & $\begin{array}{r}38.0 \\
1.0\end{array}$ & $\begin{array}{r}34.9 \\
2.0\end{array}$ \\
\hline $18: 2 n-6$ & $\begin{array}{l}\text { Mean } \\
\text { SD }\end{array}$ & $10 \cdot 3$ & $\begin{array}{r}16 \cdot 7 \\
2 \cdot 8\end{array}$ & $\begin{array}{r}13.2 \\
1.5\end{array}$ & $\begin{array}{r}11.6 \\
0.9\end{array}$ & $\begin{array}{r}13.5 \\
0.8\end{array}$ & $\begin{array}{r}15 \cdot 8 \\
5 \cdot 4\end{array}$ & $\begin{array}{r}17 \cdot 3 \\
3 \cdot 1\end{array}$ & $\begin{array}{r}13.6 \\
1.1\end{array}$ & $\begin{array}{r}14.9 \\
2.0\end{array}$ & $\begin{array}{r}13.7 \\
1.0\end{array}$ & $\begin{array}{r}15.7 \\
1.3\end{array}$ \\
\hline $18: 3 n-3$ & $\begin{array}{l}\text { Mean } \\
\text { SD }\end{array}$ & 0.7 & $\begin{array}{l}0.5 \\
0.6\end{array}$ & $\begin{array}{l}0.5 \\
0.3\end{array}$ & $\begin{array}{l}0.4 \\
0.2\end{array}$ & $\begin{array}{l}0.3 \\
0.1\end{array}$ & $\begin{array}{l}0.5 \\
0.3\end{array}$ & $\begin{array}{l}1.2^{*} \\
0.6\end{array}$ & $\begin{array}{l}0.5 \\
0.1\end{array}$ & $\begin{array}{l}0.4 \\
0.1\end{array}$ & $\begin{array}{l}0.4 \\
0.1\end{array}$ & $\begin{array}{l}0.4 \\
0.2\end{array}$ \\
\hline
\end{tabular}

Mean values were significantly different from those of the control group: ${ }^{*} P<0.05,{ }^{* *} P<0.01$.

†The sum of trans isomers.

$¥$ The sum of cis isomers.

$\S$ For details of test meals and procedures see pp. 470-471. 
Table 4. Major fatty acids in lard (LD) and chylomicrons isolated every second hour in the postprandial period ( $\mathrm{g} / 100 \mathrm{~g}$ total fatty acids)§ (Mean values and standard deviations for eight normal-weight and seven obese women)

\begin{tabular}{|c|c|c|c|c|c|c|c|c|c|c|c|c|}
\hline \multirow[b]{2}{*}{$\begin{array}{l}\text { Time (h) ... } \\
\text { Fatty acid }\end{array}$} & & \multirow[b]{2}{*}{ LD } & \multicolumn{5}{|c|}{ Normal-weight } & \multicolumn{5}{|c|}{ Obese } \\
\hline & & & 0 & 2 & 4 & 6 & 8 & 0 & 2 & 4 & 6 & 8 \\
\hline $16: 0$ & $\begin{array}{l}\text { Mean } \\
\text { SD }\end{array}$ & $25 \cdot 5$ & $\begin{array}{r}22 \cdot 3 \\
2 \cdot 4\end{array}$ & $\begin{array}{r}25.1 \\
0.9\end{array}$ & $\begin{array}{r}24 \cdot 1 \\
1.2\end{array}$ & $\begin{array}{r}24.5 \\
1.4\end{array}$ & $\begin{array}{r}22.9 \\
2.5\end{array}$ & $\begin{array}{r}24.0 \\
2.5\end{array}$ & $\begin{array}{r}25.5 \\
1.1\end{array}$ & $\begin{array}{r}23.9 \\
3.2\end{array}$ & $\begin{array}{r}24.6 \\
1.1\end{array}$ & $\begin{array}{r}24.1 \\
0.7\end{array}$ \\
\hline $16: 1 n-7$ & $\begin{array}{l}\text { Mean } \\
\text { SD }\end{array}$ & $2 \cdot 2$ & $\begin{array}{l}2 \cdot 1 \\
0.9\end{array}$ & $\begin{array}{l}2 \cdot 1 \\
0.2\end{array}$ & $\begin{array}{l}2.0 \\
0.5\end{array}$ & $\begin{array}{l}2 \cdot 0 \\
0 \cdot 1\end{array}$ & $\begin{array}{l}1.6 \\
0.3\end{array}$ & $\begin{array}{l}2.5 \\
0.7\end{array}$ & $\begin{array}{l}2 \cdot 1 \\
0.9\end{array}$ & $\begin{array}{l}1.9 \\
0.4\end{array}$ & $\begin{array}{l}2.1 \\
0.2\end{array}$ & $\begin{array}{l}2.0 \\
0.3\end{array}$ \\
\hline $18: 0$ & $\begin{array}{l}\text { Mean } \\
\text { SD }\end{array}$ & $16 \cdot 8$ & $\begin{array}{r}11.0 \\
5.3\end{array}$ & $\begin{array}{r}14.9 \\
0.8\end{array}$ & $\begin{array}{r}14.5 \\
1.2\end{array}$ & $\begin{array}{r}14.0 \\
0.5\end{array}$ & $\begin{array}{r}13.6 \\
2.0\end{array}$ & $\begin{array}{l}7.7 \\
1.5\end{array}$ & $\begin{array}{r}12.6 \\
1.7\end{array}$ & $\begin{array}{r}12 \cdot 7 \\
2.8\end{array}$ & $\begin{array}{r}12.9 \\
0.8\end{array}$ & $\begin{array}{r}12.3 \\
1.0\end{array}$ \\
\hline $18: 1$ trans $\dagger$ & $\begin{array}{l}\text { Mean } \\
\text { SD }\end{array}$ & 0.3 & $\begin{array}{l}1.0 \\
0.2\end{array}$ & $\begin{array}{l}0.5 \\
0.1\end{array}$ & $\begin{array}{l}0.6 \\
0.1\end{array}$ & $\begin{array}{l}0.5 \\
0.1\end{array}$ & $\begin{array}{l}0.6 \\
0.1\end{array}$ & $\begin{array}{l}0.6^{* *} \\
0.2\end{array}$ & $\begin{array}{l}0.4 \\
0.1\end{array}$ & $\begin{array}{l}0.5 \\
0.1\end{array}$ & $\begin{array}{l}0.5 \\
0.1\end{array}$ & $\begin{array}{l}0.5 \\
0.1\end{array}$ \\
\hline $18: 1$ cis $\ddagger$ & $\begin{array}{l}\text { Mean } \\
\text { SD }\end{array}$ & $39 \cdot 2$ & $\begin{array}{r}26 \cdot 7 \\
4.9\end{array}$ & $\begin{array}{r}36 \cdot 3 \\
1.9\end{array}$ & $\begin{array}{r}34.5 \\
2.3\end{array}$ & $\begin{array}{r}37 \cdot 1 \\
1 \cdot 1\end{array}$ & $\begin{array}{r}31.6 \\
3.7\end{array}$ & $\begin{array}{c}33.2^{*} \\
2.8\end{array}$ & $\begin{array}{r}37.7 \\
0.8\end{array}$ & $\begin{array}{r}38.4 \\
1.1\end{array}$ & $\begin{array}{r}36.7 \\
1.9\end{array}$ & $\begin{array}{r}35.2 \\
1.9\end{array}$ \\
\hline $18: 2 n-6$ & $\begin{array}{l}\text { Mean } \\
\text { SD }\end{array}$ & $10 \cdot 0$ & $\begin{array}{r}18 \cdot 7 \\
3.4\end{array}$ & $\begin{array}{r}12 \cdot 0 \\
1.3\end{array}$ & $\begin{array}{r}12.9 \\
1.4\end{array}$ & $\begin{array}{r}12 \cdot 7 \\
1.2\end{array}$ & $\begin{array}{r}13.1 \\
1.0\end{array}$ & $\begin{array}{r}17 \cdot 1 \\
3.5\end{array}$ & $\begin{array}{r}12.5 \\
0.9\end{array}$ & $\begin{array}{r}13.3 \\
1.5\end{array}$ & $\begin{array}{r}13.0 \\
1.2\end{array}$ & $\begin{array}{r}14.0 \\
2.0\end{array}$ \\
\hline $18: 3 n-3$ & $\begin{array}{l}\text { Mean } \\
\text { SD }\end{array}$ & 1.2 & $\begin{array}{l}0.5 \\
0.7\end{array}$ & $\begin{array}{l}0.9 \\
0.1\end{array}$ & $\begin{array}{l}0.9 \\
0.3\end{array}$ & $\begin{array}{l}0.9 \\
0.2\end{array}$ & $\begin{array}{l}0.6 \\
0.4\end{array}$ & $\begin{array}{l}0.8 \\
0.3\end{array}$ & $\begin{array}{l}0.9 \\
0.1\end{array}$ & $\begin{array}{l}0.8 \\
0.2\end{array}$ & $\begin{array}{l}0.8 \\
0.1\end{array}$ & $\begin{array}{l}0.8 \\
0.1\end{array}$ \\
\hline
\end{tabular}

Mean values were significantly different from those of the control group: ${ }^{*} P<0.05,{ }^{* *} P<0.01$.

$\dagger$ The sum of trans isomers.

$\ddagger$ The sum of cis isomers.

$\S$ For details of test meals and procedures see pp. 470-471.

after the test meal with PPM whereas for CM-TAG incremental AUC the difference was more distinct for LD. There was no significant difference in the incremental AUC following the three test meals for either of the two groups.

The first TAG peak in plasma and in $\mathrm{CM}\left(\mathrm{TAG}_{\mathrm{MAX}}, \mathrm{CM}-\right.$ $\mathrm{TAG}_{\mathrm{MAX}}$ respectively) was significantly higher in the obese women compared with the normal-weight women $(P<$ $0.02, P<0.01$ respectively) (Table 2 ). Neither $\mathrm{TAG}_{\mathrm{MAX}}$ nor CM-TAG $\mathrm{GAX}_{\mathrm{MA}}$ were significantly different when comparing test meals. The time to the first maximum postprandial plasma total TAG concentration (TAG $\mathrm{T}_{\mathrm{MAX}}$ ) and CM-TAG concentration (CM-TAG T $_{\text {MAX }}$ ) was not significantly different between groups or test meals.

\section{Fatty acid composition of chylomicrons}

The fatty acid compositions of the different fats used in the present study together with the compositions of their respective $\mathrm{CM}$ isolated every second hour are shown in Tables 3, 4 and 5. The fatty acid compositions in the fasting state were similar for all $3 \mathrm{~d}$ of treatment. The overall fatty acid composition was not very different between the two

Table 5. Major fatty acids in puff-pastry margarine (PPM) and chylomicrons isolated every second hour in the postprandial period ( $\mathrm{g} / 100 \mathrm{~g}$ total fatty acids)§

(Mean values and standard deviations for eight normal-weight and seven obese women)

\begin{tabular}{|c|c|c|c|c|c|c|c|c|c|c|c|c|}
\hline \multirow[b]{2}{*}{$\begin{array}{l}\text { Time (h) ... } \\
\text { Fatty acid }\end{array}$} & & \multirow[b]{2}{*}{ PPM } & \multicolumn{5}{|c|}{ Normal-weight } & \multicolumn{5}{|c|}{ Obese } \\
\hline & & & 0 & 2 & 4 & 6 & 8 & 0 & 2 & 4 & 6 & 8 \\
\hline $16: 0$ & $\begin{array}{l}\text { Mean } \\
\text { SD }\end{array}$ & 34.5 & $\begin{array}{r}23 \cdot 7 \\
1.6\end{array}$ & $\begin{array}{r}31.0 \\
0.7\end{array}$ & $\begin{array}{r}29 \cdot 8 \\
1.0\end{array}$ & $\begin{array}{r}30.1 \\
0.9\end{array}$ & $\begin{array}{r}27 \cdot 8 \\
1.2\end{array}$ & $\begin{array}{r}23 \cdot 0 \\
4 \cdot 1\end{array}$ & $\begin{array}{r}29 \cdot 7 \\
1 \cdot 8\end{array}$ & $\begin{array}{r}29 \cdot 9 \\
1.4\end{array}$ & $\begin{array}{r}29 \cdot 3 \\
2 \cdot 3\end{array}$ & $\begin{array}{r}26.9 \\
1.7\end{array}$ \\
\hline $16: 1 n-7$ & $\begin{array}{l}\text { Mean } \\
\text { SD }\end{array}$ & $0 \cdot 1$ & $\begin{array}{l}2 \cdot 4 \\
1 \cdot 1\end{array}$ & $\begin{array}{l}0.6 \\
0.2\end{array}$ & $\begin{array}{l}0.7 \\
0.2\end{array}$ & $\begin{array}{l}0.6 \\
0.2\end{array}$ & $\begin{array}{l}0.9 \\
0.2\end{array}$ & $\begin{array}{l}3 \cdot 1 \\
1 \cdot 7\end{array}$ & $\begin{array}{l}1.2 \\
0.9\end{array}$ & $\begin{array}{l}1.2 \\
0.9\end{array}$ & $\begin{array}{l}1 \cdot 2 \\
0.8\end{array}$ & $\begin{array}{l}1.9 \\
0.9\end{array}$ \\
\hline $18: 0$ & $\begin{array}{l}\text { Mean } \\
\text { SD }\end{array}$ & $7 \cdot 4$ & $\begin{array}{l}8 \cdot 8 \\
1 \cdot 3\end{array}$ & $\begin{array}{l}8 \cdot 1 \\
0 \cdot 4\end{array}$ & $\begin{array}{l}8.5 \\
0.3\end{array}$ & $\begin{array}{l}8.2 \\
0.5\end{array}$ & $\begin{array}{l}6.7 \\
0.5\end{array}$ & $\begin{array}{l}7 \cdot 0 \\
2 \cdot 3\end{array}$ & $\begin{array}{l}7.6 \\
0.9\end{array}$ & $\begin{array}{l}7.7 \\
0.6\end{array}$ & $\begin{array}{l}7 \cdot 6 \\
0.7\end{array}$ & $\begin{array}{l}7.5 \\
1.4\end{array}$ \\
\hline $18: 1$ trans $\dagger$ & $\begin{array}{l}\text { Mean } \\
\text { SD }\end{array}$ & $7 \cdot 0$ & $\begin{array}{l}0.8 \\
0.3\end{array}$ & $\begin{array}{l}5 \cdot 3 \\
0.3\end{array}$ & $\begin{array}{l}4.9 \\
0.4\end{array}$ & $\begin{array}{l}5 \cdot 1 \\
0.6\end{array}$ & $\begin{array}{l}3.7 \\
0.8\end{array}$ & $\begin{array}{l}0.7 \\
0.4\end{array}$ & $\begin{array}{l}4.5 \\
0.6\end{array}$ & $\begin{array}{l}4.5 \\
0.6\end{array}$ & $\begin{array}{l}4 \cdot 3 \\
0 \cdot 8\end{array}$ & $\begin{array}{l}2.9 \\
0.8\end{array}$ \\
\hline $18: 1$ cis $\ddagger$ & $\begin{array}{l}\text { Mean } \\
\text { SD }\end{array}$ & $31 \cdot 5$ & $\begin{array}{r}28.5 \\
4.8\end{array}$ & $\begin{array}{r}31.6 \\
0.7\end{array}$ & $\begin{array}{r}30 \cdot 2 \\
1.2\end{array}$ & $\begin{array}{r}29.9 \\
2.5\end{array}$ & $\begin{array}{r}26.9 \\
3.0\end{array}$ & $\begin{array}{c}35 \cdot 5^{\star} \\
3.8\end{array}$ & $\begin{array}{r}33.3 \\
1.0\end{array}$ & $\begin{array}{r}33.0 \\
0.6\end{array}$ & $\begin{array}{r}32.6 \\
1.2\end{array}$ & $\begin{array}{r}31.5 \\
2.7\end{array}$ \\
\hline $18: 2 n-6$ & $\begin{array}{l}\text { Mean } \\
\text { SD }\end{array}$ & $14 \cdot 5$ & $\begin{array}{r}17 \cdot 2 \\
2 \cdot 6\end{array}$ & $\begin{array}{r}15.9 \\
0.7\end{array}$ & $\begin{array}{r}16.5 \\
1.0\end{array}$ & $\begin{array}{r}16 \cdot 6 \\
0.9\end{array}$ & $\begin{array}{r}18.2 \\
1.6\end{array}$ & $\begin{array}{r}16 \cdot 5 \\
2.8\end{array}$ & $\begin{array}{r}16 \cdot 0 \\
1.6\end{array}$ & $\begin{array}{r}15 \cdot 6 \\
1 \cdot 2\end{array}$ & $\begin{array}{r}16 \cdot 4 \\
1.7\end{array}$ & $\begin{array}{r}18.0 \\
2.7\end{array}$ \\
\hline $18: 3 n-3$ & $\begin{array}{l}\text { Mean } \\
\text { SD }\end{array}$ & $2 \cdot 1$ & $\begin{array}{l}0.8 \\
0.8\end{array}$ & $\begin{array}{l}1.4 \\
0.2\end{array}$ & $\begin{array}{l}1.3 \\
0.3\end{array}$ & $\begin{array}{l}1 \cdot 2 \\
0.2\end{array}$ & $\begin{array}{l}1 \cdot 0 \\
0.1\end{array}$ & $\begin{array}{l}1.4 \\
1.0\end{array}$ & $\begin{array}{l}1.5 \\
0.3\end{array}$ & $\begin{array}{l}1 \cdot 3 \\
0.2\end{array}$ & $\begin{array}{l}1 \cdot 2 \\
0.2\end{array}$ & $\begin{array}{l}1.0 \\
0.4\end{array}$ \\
\hline
\end{tabular}

Mean values were significantly different from those of the control group: * $P<0.05$.

$\dagger$ The sum of trans isomers.

$\ddagger$ The sum of cis isomers.

$\S$ For details of test meals and procedures see pp. 470-471. 

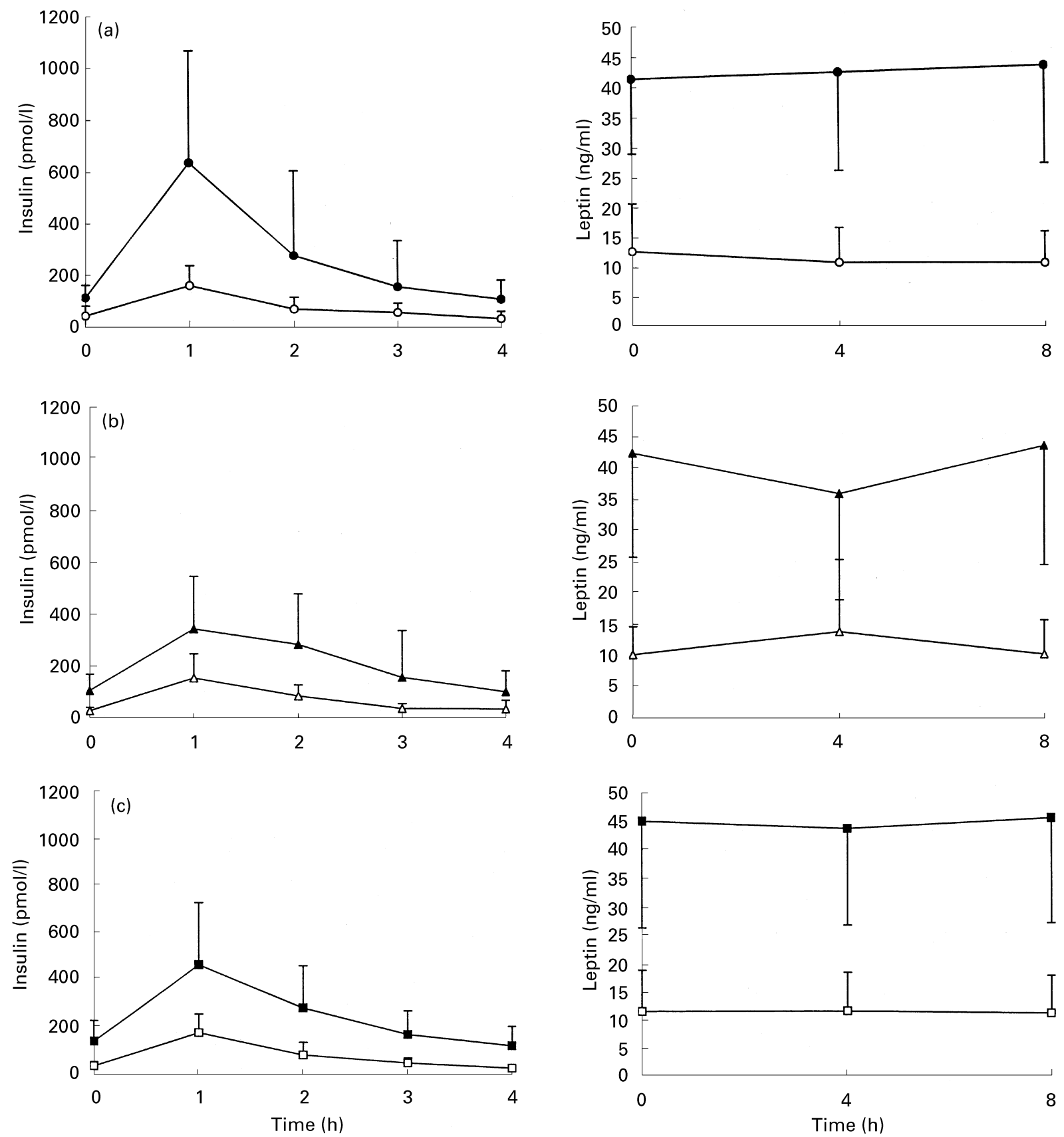

Fig. 2. Insulin responses $(0-4 \mathrm{~h})$ and concentrations of leptin $(0,4$ and $8 \mathrm{~h})$ after test meals containing (a) palm oil $(O, \bullet)$, (b) lard $(\triangle$, $\mathbf{\Delta})$, and (c) puff-pastry margarine $(\square, \boldsymbol{\square})$ in normal-weight $(\bigcirc, \Delta, \square)$ and obese $(\bullet, \mathbf{\Lambda}, \mathbf{\square})$ women. Values are means with standard deviations represented by vertical bars. For details of test meals and procedures see pp. 470-471.

groups, however, the obese women had significantly higher contents of cis 18:1 isomers compared with the normalweight women $(P<0 \cdot 05)$. The fasting values of $18: 2 n-6$ were relatively high for both groups and not significantly different. In the postprandial period the fatty acid composition of CM lipids reflected that of dietary fat and particularly so at the maximal absorption $(2 \mathrm{~h})$.

\section{Cholesterol}

There was no statistically significant difference between the obese and the normal-weight women for fasting total $\mathrm{CH}$
(4.6 (SD 0.9) v. $4.3(\mathrm{SD} 0.2) \mathrm{mmol} / \mathrm{l}$ respectively) or LDL$\mathrm{CH}(2.8$ (SD 0.6) v. 2.2 (SD 0.3$)$ mmol/l respectively). However, fasting HDL-CH was significantly lower in the obese women compared with the normal-weight women $(1 \cdot 2(\mathrm{SD} 0 \cdot 1)$ v. $1 \cdot 7(\mathrm{SD} 0 \cdot 1) \mathrm{mmol} / \mathrm{l}$ respectively, $P<0 \cdot 01)$.

\section{Insulin and leptin}

Table 2 presents the fasting concentrations and postprandial responses of insulin and leptin in normal-weight and obese women. The fasting insulin concentrations were significantly higher in the obese women than in the 
normal-weight women $(P<0 \cdot 001)$. The insulin concentrations increased significantly at $1 \mathrm{~h}$ in both groups and returned to baseline at 3-4h (Fig. 2). The incremental areas under the insulin response curves were significantly higher in the obese women compared with the normalweight women $(P<0.001)$ (Table 2$)$. However, there was no significant difference in the incremental AUC following the three test meals for either of the two groups. The fasting leptin concentrations were significantly higher in the obese group than in the normal-weight group $(P<0 \cdot 001)$ (Table 2) but there were no significant postprandial changes in the concentrations of leptin (Fig. 2).

\section{Discussion}

Information about the acute effect on lipaemia is sparse in obese subjects after consumption of different types of fat. Most previous studies have focused on postprandial insulinaemia and glycaemia (Rasmussen et al. 1996; Christiansen et al. 1997). Our results indicate that test meals containing PO, LD, or PPM produce similar postprandial responses of plasma total TAG and CM-TAG in both normal-weight and obese young women, although the differences in the fatty acid composition of the fats were distinct. However, we cannot exclude that even more marked differences in the fatty acid pattern would have resulted in different postprandial responses.

The amount of fat administered was related to the subject's body surface area; thus, generally, the obese women consumed more fat than the normal-weight women (51 (SD 4) and 64 (SD 6) g per meal respectively). The reason for this was based on an earlier study of Griffin et al. (1945) who found a slightly better correlation between plasma volume and body surface area compared with either weight or height. The obese women had significantly higher postprandial responses of TAG than the normal-weight women. This supports earlier investigations of obesity where body surface area also was used to determine the amount of administered fat (Lewis et al. 1990, 1991; Couillard et al. 1998). In studies of obesity where all subjects were fed on the same amount of fat the obese subjects still displayed greater elevations in TAG concentrations in the postprandial period (Coppack et al. 1992; Potts et al. 1994, 1995).

In agreement with previous studies fasting TAG concentrations were significantly higher in the obese compared with the normal-weight women (Lewis et al. 1990; Potts et al. 1995; Couillard et al. 1998). In some of the earlier studies the magnitude of the postprandial TAG responses was found to be closely related to the fasting TAG concentrations (Lewis et al. 1990, 1991; O'Meara et al. 1992; Potts et al. 1994; Couillard et al. 1998), but this association was not found to be significant in the present study. However, the fasting TAG concentrations in this study, as in others (Lewis et al. 1990; Akanji et al. 1992; O'Meara et al. 1992; Potts et al. 1994; Couillard et al. 1998) were related to the obesity of the women.

The pattern of postprandial TAG varies considerably between subjects (Cohn et al. 1988b); plasma total TAG may be found to peak either once, twice, or three times during the postprandial period. The majority of the women in the present study showed a biphasic response curve. The nearly fat-free $(<1 \mathrm{~g}$ fat) lunch after $4 \mathrm{~h}$ could have influenced the response curves of TAG. This is based on the study of Ercan et al. (1994) who investigated whether fat ingestion with a morning meal had an effect on TAG responses to a second meal not containing fat, and observed a biphasic curve. The two incremental AUC $(0-4 \mathrm{~h}$ and $4-8 \mathrm{~h}$ respectively) were similar. If fat was present in the second meal the areas were even greater. Nevertheless, no increase in the postprandial TAG concentrations in plasma or lipoprotein fractions was seen after ingestion of $5 \mathrm{~g}$ fat (Jeppesen et al. 1995). The rise in plasma total TAG corresponded to marked increases in the TAG concentrations of $\mathrm{CM}$ and a positive correlation between the postprandial areas was found $(r 0.79 ; P<0.05)$.

The difference in the level of plasma total TAG and CMTAG represents the contribution of TAG from other lipoproteins especially VLDL with minor contributions from LDL and HDL (Potts et al. 1994). VLDL is the major lipoprotein present in the fasting state and contaminations of the CM fractions with VLDL have been revealed by combining centrifugation with SDS-polyacrylamide gel electrophoresis (Potts et al. 1994). A contamination would explain the higher fasting concentrations of CM-TAG in the obese women and in addition the fatty acid composition of the CM lipids. Probably the higher VLDL levels in the obese women (results not shown) are due to an increased hepatic VLDL production despite higher fasting insulin levels, i.e. reflecting a higher degree of insulin resistance. It was notable that the obese women had significantly higher contents of cis 18:1 isomers than the normal-weight women. These differences between the two groups could be a result of different food intake the day before the study or a contamination of VLDL particles. The fatty acid composition of the fasting VLDL fractions (results not shown) showed low contents of $18: 0$ (approximately $5 \mathrm{~g} / 100 \mathrm{~g}$ total fatty acids) and high contents of cis 18:1 isomers (approximately $34 \mathrm{~g} / 100 \mathrm{~g}$ total fatty acids) in both groups. However, the fasting fatty acid compositions for normal-weight and obese women were surprisingly equal, indicating that the fats consumed by the obese women are not characterized by high amounts of the more atherogenic SFA and correspondingly lower levels of PUFA. The obesity is therefore not related to differences in oxidative degradation of ingested fatty acids.

The fatty acid pattern after the meals changed during the first $2 \mathrm{~h}$; thus the composition of CM lipids reflected that of the dietary fat ingested at peak absorption $(2 \mathrm{~h})$. This corroborates with previous postprandial studies of compositional changes of CM lipids in human subjects (Bonanome \& Grundy, 1989; Nestel et al. 1995) and in rats (Lambert et al. 1996). However, no changes appeared for either of the two groups through the postprandial period $(2-6 \mathrm{~h})$, indicating that no preferential clearing of TAG occurred. The fatty acid compositions gradually approached the fasting values when the CM-TAG concentrations declined after 7-8 h. The obese women had significantly lower concentrations of HDL-CH than the normal-weight women, which is in agreement with earlier findings (Lewis et al. 1990; Couillard et al. 1998). As the total $\mathrm{CH}$ is equal for the two groups, the lower HDL-CH was probably compensated for by a higher amount of $\mathrm{CH}$ in the VLDL fraction. Despres et al. 
(1987) suggested that adipose cells, particularly enlarged cells from abdominal subcutaneous fat in obesity, could be directly responsible for the decreased plasma HDL-CH concentrations due to increased uptake of $\mathrm{CH}$.

As expected, the fasting concentrations of insulin and the postprandial response areas were significantly higher in the obese group than in the normal-weight group but there was no statistically significant difference between the three test meals. Gatti et al. (1992) reported similar insulin responses in normal volunteers after ingestion of saturated (butter) and unsaturated fatty acids (olive and maize oil) compared with bread alone. In contrast, Rasmussen et al. (1996) reported elevated insulin concentrations in patients with non-insulin-dependent diabetes mellitus after ingestion of mashed potato with saturated fat (butter) compared with potato alone, whereas addition of monounsaturated oil (olive oil) apparently had no effect. The effect of butter agrees with findings of Gannon et al. (1993) and Collier et al. (1988). However, the studies mentioned used a meal without fat as reference.

Recent studies have documented an increased level of serum leptin in obese human subjects (Hamilton et al. 1995; Lönnqvist et al. 1995; Maffei et al. 1995; Considine et al. $1996 b$ ). These observations are consistent with the results of the present study where we found a four-times higher level of leptin in the obese group compared with the normal-weight group and strong positive correlations between serum leptin concentrations and BMI and fasting insulin concentrations ( $r 0.80 ; P<0.01$ and $r 0.89 ; P<0.01$ respectively). As the $o b$ gene is normally expressed in adipocytes of obese subjects and as the coding sequence of $o b$ gene is not altered in obese subjects (Considine et al. 1995, 1996a), obese subjects appear to be resistant to their endogenous leptin. A number of studies indicate that leptin levels increase 5-10 h after meals (Kolaczynski et al. 1996b; Malmström et al. 1996; Laughlin et al. 1997; Schoeller et al. 1997; Saad et al. 1998). However, most studies have been unable to detect that the increase in insulin secondary to feeding does increase serum leptin (Kolaczynski et al. 1996a; Muscelli et al. 1996; Pratley et al. 1996; Ryan \& Elahi, 1996; Vidal et al. 1996). The present data corroborate the opinion that metabolic regulation of leptin secretion most probably occurs at the level of transcription and is not due to acutely regulated secretory pathways. Furthermore, it is in agreement with the study of Joannic et al. (1998) showing no effect of changing the content of fatty acids from MUFA to PUFA in the test meals.

In summary, our results clearly demonstrate that obese women have exaggerated postprandial lipid and hormone responses compared with those of normal-weight women. The different fatty acid compositions of normal dietary fats such as PO, LD, and PPM show no influence on the measured postprandial responses. The unchanged fatty acid profiles during the postprandial period indicate that neither discrimination in the absorption nor any preferential clearing took place.

\section{References}

Akanji A, Nzegwu AA \& Agbedana EO (1992) Some determinants of postprandial lipaemia in Nigerian diabetic and non-diabetic subjects. British Journal of Nutrition 68, 153-162.
Alstrup KK, Gregersen S, Jensen HM, Thomsen JL \& Hermansen K (1999) Differential effects of cis and trans fatty acids on insulin release from isolated mouse islets. Metabolism 48, 22-29.

Andersen L, Dinesen B, Jørgensen PN, Poulsen F \& Røder ME (1993) Enzyme immunoassay for intact human insulin in serum and plasma. Clinical Chemistry 39, 578-582.

Bonanome A \& Grundy SM (1988) Effect of dietary stearic acid on plasma cholesterol and lipoprotein levels. New England Journal of Medicine 318, 1244-1248.

Bonanome A \& Grundy SM (1989) Intestinal absorption of stearic acid after consumption of high fat meals in humans. Journal of Nutrition 119, 1556-1560.

Brunzell JD, Hazzard WR, Porte D \& Bierman EL (1973) Evidence for a common, saturable, triglyceride removal mechanism for chylomicrons and very low density lipoproteins in man. Journal of Clinical Investigation 52, 1578-1585.

Christiansen E, Schnider S, Palmvig B, Tauber-Lassen E \& Pedersen O (1997) Intake of a diet high in trans monounsaturated fatty acids or saturated fatty acids. Diabetes Care 20, 881-887.

Cohn JS, McNamara JR, Cohn SD, Ordovas JM \& Schaefer EJ (1988a) Plasma apolipoprotein changes in the triglyceride-rich lipoprotein fraction of human subjects fed a fat-rich meal. Journal of Lipid Research 29, 925-936.

Cohn JS, McNamara JR, Cohn SD, Ordovas JM \& Schaefer EJ (1988b) Postprandial plasma lipoprotein changes in human subjects of different ages. Journal of Lipid Research 29, 469-479.

Collier GR, Greenberg GR, Wolever TMS \& Jenkins DJA (1988) The acute effect of fat on insulin secretion. Journal of Clinical Endocrinology and Metabolism 66, 323-326.

Considine RV, Considine EL, Williams CJ, Nyce MR, Magosin SA, Bauer TL, Rosato EL, Colberg J \& Caro JF (1995) Evidence against either a premature stop codon or the absence of obese gene mRNA in human obesity. Journal of Clinical Investigation 95, 2986-2988.

Considine RV, Considine EL, Williams CJ, Nyce MR, Zhang P, Opentanova I, Ohannesian JP, Kolaczynski JW, Bauer TL, Moore JH \& Caro JF (1996a) Mutation screening and identification of a sequence variation in the human $o b$ gene coding region. Biochemical and Biophysical Research Communications 220, 735-739.

Considine RV, Sinha MK, Heiman ML, Kriauciunas A, Stephens TW, Nyce MR, Ohannesian JP, Marco CC, McKee LJ, Bauer TL \& Caro JF (1996b) Serum immunoreactive-leptin concentrations in normal-weight and obese humans. New England Journal of Medicine 334, 292-295.

Coppack SW, Evans RD, Fisher RM, Frayn KN, Gibbons GF, Humphreys SM, Kirk MJ, Potts JL \& Hockaday TDR (1992) Adipose tissue metabolism in obesity: lipase action in vivo before and after a mixed meal. Metabolism 41, 264-272.

Couillard C, Bergeron N, Prud'homme D, Bergeron J, Tremblay A, Bouchard C, Mauriège P \& Despres J-P (1998) Postprandial triglyceride response in visceral obesity in men. Diabetes $\mathbf{4 7}$, 953-960.

Coulston AM, Hollenbeck CB, Swislocki ALM \& Reaven GM (1989) Persistence of hypertriglyceridemic effect of low-fat highcarbohydrate diets in NIDDM patients. Diabetes Care 12, 94-101.

Denke MA \& Grundy SM (1992) Comparisons of effects of lauric acid and palmitic acid on plasma lipids and lipoproteins. American Journal of Clinical Nutrition 56, 895-898.

Despres J-P, Fong BS, Julien P, Jimenez J \& Angel A (1987) Regional variation in HDL metabolism in human fat cells: effect of cell size. American Journal of Physiology 252, E654-E659.

Ercan N, Gannon MC \& Nuttall FQ (1994) Effect of added fat on the plasma glucose and insulin response to ingested potato given in various combinations as two meals in normal individuals. Diabetes Care 17, 1453-1459. 
Friedewald WT, Levy RI \& Fredrickson DS (1972) Estimation of the concentration of low-density lipoprotein cholesterol in plasma without use of the preparative ultracentrifuge. Clinical Chemistry 18, 499-502.

Gannon MC, Nuttall FQ, Westphal SA \& Seaquist ER (1993) The effect of fat and carbohydrate on plasma glucose, insulin, Cpeptide, and triglycerides in normal male subjects. Journal of the American College of Nutrition 12, 36-41.

Garg A, Bonanome A, Grundy SM, Zhang ZJ \& Unger RH (1988) Comparison of a high-carbohydrate diet with a high-monounsaturated-fat diet in patients with non-insulin-dependent diabetes mellitus. New England Journal of Medicine 319, 829-834.

Garg A, Grundy SM \& Unger RH (1992) Comparison of effects of high and low carbohydrate diets on plasma lipoproteins and insulin sensitivity in patients with mild NIDDM. Diabetes $\mathbf{4 1}$, $1278-1285$.

Gatti E, Noè D, Pazzucconi F, Gianfranceschi G, Porrini M, Testolin G \& Sirtori CR (1992) Differential effect of unsaturated oils and butter on blood glucose and insulin response to carbohydrate in normal volunteers. European Journal of Clinical Nutrition 46, 161-166.

Grande F, Anderson JT \& Keys A (1970) Comparison of effects of palmitic and stearic acids in the diet on serum cholesterol in man. American Journal of Clinical Nutrition 23, 1184-1193.

Griffin GE, Abbott WE, Pride MP, Muntwyler E, Mautz FR \& Griffith L (1945) Plasma volume, "available (thiocyanate) volume" and total circulating plasma proteins in normal adults. Annals of Surgery 121, 352-360.

Groot PHE, van Stiphout W-AHJ, Krauss XH, Jansen H \& van Tol A (1991) Postprandial lipoprotein metabolism in normolipidemic men with and without coronary artery disease. Arteriosclerosis and Thrombosis 11, 653-662.

Hamilton BS, Paglia D, Kwan AYM \& Deitel M (1995) Increased obese mRNA expression in omental fat cells from massively obese humans. Nature Medicine 1, 953-956.

Hegsted DM, McGandy RB, Myers ML \& Stare FJ (1965) Quantitative effects of dietary fat on serum cholesterol in man. American Journal of Clinical Nutrition 17, 281-295.

Jeppesen J, Chen, Y-DI, Zhou M-Y, Wang T \& Reaven GM (1995) Effect of variations in oral fat and carbohydrate load on postprandial lipemia. American Journal of Clinical Nutrition 62, 1201-1205.

Joannic J-L, Oppert J-M, Lahlou N, Basdevant A, Auboiron S, Raison J, Bornet F \& Guy-Grand B (1998) Plasma leptin and hunger ratings in healthy humans. Appetite 30, 129-138.

Judd JT, Clevidence BA, Muesing RA, Wittes J, Sunkin ME \& Podczasy JJ (1994) Dietary trans fatty acids: effects on plasma lipids and lipoproteins of healthy men and women. American Journal of Clinical Nutrition 59, 861-868.

Karpe F, Steiner G, Uffelman K, Olivecrona T \& Hamsten A (1994) Postprandial lipoproteins and progression of coronary atherosclerosis. Atherosclerosis 106, 83-97.

Kolaczynski JW, Nyce MR, Considine RV, Boden G, Nolan JJ, Henry R, Mudaliar SR, Olefsky J \& Caro JF (1996a) Acute and chronic effect of insulin on leptin production in humans. Studies in vivo and in vitro. Diabetes 45, 699-701.

Kolaczynski JW, Ohannesian JP, Considine RV, Marco C \& Caro JF (1996b) Responses of leptin to short-term and prolonged overfeeding in humans. Journal of Clinical Endocrinology and Metabolism 81, 4162-4165.

Lambert MS, Botham KM \& Mayes PA (1996) Modification of the fatty acid composition of dietary oils and fats on incorporation into chylomicrons and chylomicron remnants. British Journal of Nutrition 76, 435-445.

Laughlin GA \& Yen SSC (1997) Hypoleptinemia in women athletes: absence of a diurnal rhythm with amenorrhea. Journal of Clinical Endocrinology and Metabolism 82, 318-321.
Lewis GF, O’Meara NM, Soltys PA, Balckman JD, Iverius PH, Druetzler AF, Getz GS \& Polonsky KS (1990) Postprandial lipoprotein metabolism in normal and obese subjects: comparison after the vitamin A fat-loading test. Journal of Clinical Endocrinology and Metabolism 71, 1041-1050.

Lewis GF, O’Meara NM, Soltys PA, Balckman JD, Iverius PH, Pugh WL, Getz GS \& Polonsky KS (1991) Fasting hypertriglyceridemia in noninsulin-dependent diabetes mellitus is an important predictor of postprandial lipid and lipoprotein abnormalities. Journal of Clinical Endocrinology and Metabolism 72, 934-944.

Lichtenstein AH, Ausman LM, Carrasco W, Jenner JL, Ordovas JM \& Schaefer EJ (1993) Hydrogenation impairs the hypolipidemic effect of corn oil in humans. Arteriosclerosis and Thrombosis 13, 154-161.

Lönnqvist F, Arner P, Nordfors L \& Schalling M (1995) Overexpression of the obese $(o b)$ gene in adipose tissue of human obese subjects. Nature Medicine 1, 950-953.

Maffei M, Halaas J, Ravussin E, Pratley RE, Lee GH, Zhang Y, Fei H, Kim S, Lallone R, Ranganathan S, Kern PA \& Friedman JM (1995) Leptin levels in human and rodent: measurement of plasma leptin and $o b$ RNA in obese and weight-reduced subjects. Nature Medicine 1, 1155-1161.

Malmström R, Taskinen M-R, Karonen S-L \& Yki-Jarvinen H (1996) Insulin increases plasma leptin concentrations in normal subjects and patients with NIDDM. Diabetologia 39, 993-996.

Matthews JNS, Altman DG, Campbell MJ \& Royston P (1990) Analysis of serial measurements in medical research. British Medical Journal 300, 230-235.

Mensink RP, Zock PL, Katan MB \& Hornstra G (1992) Effect of dietary cis and trans fatty acids on serum lipoprotein(a) levels in humans. Journal of Lipid Research 33, 1493-1501.

Muscelli E, Camastra S, Masoni A, Baldi S, Sironi AM, Natali A \& Ferrannini E (1996) Acute insulin administration does not affect plasma leptin levels in lean or obese subjects. European Journal of Clinical Investigation 26, 940-943.

Nestel PJ, Noakes M, Belling GB, McArthur R \& Clifton PM (1995) Effect on plasma lipids of interesterifying a mix of edible oils. American Journal of Clinical Nutrition 62, 950-955.

Nestel P, Noakes M, Belling B, McArthur R, Clifton P, Janus E \& Abbey M (1992) Plasma lipoprotein lipid and Lp(a) changes with substitution of elaidic acid for oleic acid in the diet. Journal of Lipid Research 33, 1029-1036.

O'Meara NM, Lewis GF, Cabana VG, Iverius PH, Getz GS, \& Polonsky KS (1992) Role of basal triglyceride and high density lipoprotein in determination of postprandial lipid and lipoprotein responses. Journal of Clinical Endocrinology and Metabolism 75, 465-471.

Patsch JR, Miesenböck G, Hopferwieser T, Mühlberger V, Knapp E, Dunn JK, Gotto AM \& Patsch W (1992) Relation of triglyceride metabolism and coronary artery disease. Studies in the postprandial state. Arteriosclerosis and Thrombosis 12, 1336-1345.

Potts JL, Coppack SW, Fisher RM, Humphreys SM, Gibbons GF \& Frayn KN (1995) Impaired postprandial clearance of triacylglycerol-rich lipoproteins in adipose tissue in obese subjects. American Journal of Physiology 268, E588-E594.

Potts JL, Fisher RM, Humphreys SM, Coppack SW, Gibbons GF \& Frayn KN (1991) Peripheral triacylglycerol extraction in the fasting and post-prandial states. Clinical Science 81, 621-626.

Potts JL, Humphreys SM, Coppack SW, Fisher RM, Gibbons GF \& Frayn KN (1994) Fasting plasma triacylglycerol concentrations predict adverse changes in lipoprotein metabolism after a normal meal. British Journal of Nutrition 72, 101-109.

Pratley RE, Nicolson M, Bogardus C \& Ravussin E (1996) Effects of acute hyperinsulinaemia on plasma leptin concentrations in insulin-sensitive and insulin-resistant Pima Indians. 
Journal of Clinical Endocrinology and Metabolism 81, 44184421.

Rasmussen O, Lauszus FF, Christiansen C, Thomsen C \& Hermansen K (1996) Differential effects of saturated and monounsaturated fat on blood glucose and insulin responses in subjects with non-insulin-dependent diabetes mellitus. American Journal of Clinical Nutrition 63, 249-253.

Rasmussen OW, Thomsen C, Hansen KW, Vesterlund M, Winther E \& Hermansen K (1993) Effects on blood pressure, glucose, and lipid levels of a high-monounsaturated fat diet compared with a high-carbohydrate diet in NIDDM subjects. Diabetes Care 16, 1565-1571.

Ryan AS \& Elahi D (1996) The effects of acute hyperglycaemia and hyperinsulinaemia on plasma leptin levels: its relationship with body fat, visceral adiposity, and age in women. Journal of Clinical Endocrinology and Metabolism 81, 4433-4438.

Saad MF, Khan A, Sharma A, Michael A, Riad-Gabriel G, Boyadjian R, Jinagouda SD, Steil GM \& Kamdar V (1998) Physiological insulinemia acutely modulates plasma leptin. Diabetes 47, 544-549.

Schneeman BO, Kotite L, Todd KM \& Havel RJ (1993) Relationship between the responses of triglyceride-rich lipoproteins in blood plasma containing apolipoproteins B-48 and B-100 to a fat-containing meal in normolipidemic humans. Proceedings of the National Academy of Sciences USA 90, 2069-2073.

Schoeller DA, Cella LK, Sinha MK \& Caro JF (1997) Entrainment of the diurnal rhythm of plasma leptin to meal timing. Journal of Clinical Investigation 100, 1882-1887.

Storm H, Thomsen C, Pedersen E, Rasmussen O, Christiansen C \& Hermansen K (1997) Comparison of a carbohydrate-rich diet and diets rich in stearic or palmitic acid in NIDDM patients. Diabetes Care 20, 1807-1813.

Vidal H, Auboeuf D, De Vos P, Staels B, Riou JP, Auwerx J \& Laville M (1996) The expression of $o b$ gene is not acutely regulated by insulin and fasting in human abdominal subcutaneous adipose tissue. Journal of Clinical Investigation 98, 251255.

Willett WC, Stampfer MJ, Manson JE, Colditz GA, Speizer FE, Rosner BA, Sampson LA \& Hennekens CH (1993) Intake of trans fatty acids and risk of coronary heart disease among women. Lancet 341, 581-588.

Wood R, Kubena K, O’Brian B, Tseng S \& Martin G (1993) Effect of butter, mono- and polyunsaturated fatty acid-enriched butter, trans fatty acid margarine, and zero trans fatty acid margarine on serum lipids and lipoproteins in healthy men. Journal of Lipid Research 34, 1-11. 